\title{
A New Field Emission Scanning Electron Microscopy Facility with STEM and EDS Capabilities for Interdisciplinary Research and Education at Towson University, Fisher College of Science and Mathematics
}

\author{
Vonnie D.C. Shields ${ }^{1}$, Mary S. Devadas ${ }^{2}$, Rajeswari M. Kolagani ${ }^{3}$, Vera N. Smolyaninova ${ }^{3}$, Amy J. \\ Williams ${ }^{3}$ \\ 1. Biological Sciences Department, Fisher College of Science and Mathematics, Smith Hall, Towson \\ University, Towson, MD 21252, USA. \\ 2. Department of Chemistry, Fisher College of Science and Mathematics, Smith Hall, Towson \\ University, Towson, MD 21252, USA. \\ 3. Physics, Astronomy, and Geosciences, Fisher College of Science and Mathematics, Smith Hall, \\ Towson University, Towson, MD 21252, USA.
}

Recently, Towson University (TU) was successful in acquiring a new state-of-the-art Thermo Fisher Scientific APREO LoVac Schottky Field Emission Gun (FEG) Scanning Electron Microscope (SEM). This microscope is a high-performance SEM that combines high- and low-voltage ultra-high resolution $(\sim 1 \mathrm{~nm})$ capabilities with an electrostatic lens design. In addition, it features beam deceleration and unique in-lens detection providing unprecedented contrast and versatility of samples for research, education, and training activities across multiple disciplines, in the Fisher College of Science and Mathematics (FCSM). The instrument uses an entirely oil-free vacuum system and is standardly

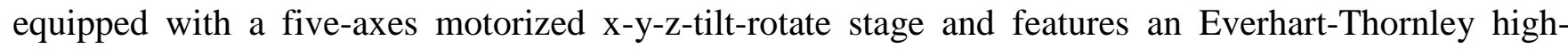
vacuum secondary electron detector (SED) optimized for use across the available $\mathrm{kV}$, current, and pressure range. It bears a Trinity detection system, comprised of a lower in-lens detector (T1), upper inlens detector (T2), and in-column detector (T3). The microscope includes a dedicated low-vacuum (LV) SED to provide charge-free topographic contrast imaging of non-conductive samples and offers an immersion lens, providing an additional magnetic objective lens. This lens, when combined with the electrostatic column, creates a compound final lens, which boosts the low-kV resolution performance to $1.0 \mathrm{~nm}$ at $1 \mathrm{kV}$ without requiring beam deceleration. The Directional Back-Scatter (DBS) detector enables separate detection of electrons emitted at different angles. This instrument comes with dispersive X-ray Spectroscopy (EDS) and Scanning Transmission Electron Microscopy (S-TEM) capabilities as well.

The acquisition of this microscope in our college enables new and ongoing multidisciplinary research projects involving faculty in the Biological Sciences, Physics, Astronomy, and Geosciences and Chemistry departments and thesis and non-thesis Master's and undergraduate students, from these departments (Fig. 1). This microscope will offer education and training benefits, in-house, to both undergraduate and graduate students, which will prove invaluable in their education, research, and professional careers in the Science, Technology, Engineering, and Mathematics (STEM) workforce. The incorporation of this instrument into several courses will truly benefit the students, whether it is to offer opportunities for hands-on experience in advanced graduate courses or as a demonstration tool in courses offering sample preparation level experience in lower level courses. As field emission scanning electron microscopic instrumentation is increasingly common in private and governmental laboratories, acquisition of such an instrument and its incorporation into the teaching curriculum will ensure highly knowledgeable, skilled, and experienced individuals in both the regional and national workforces. Research, education and training opportunities, facilitated by this instrument will increase the 
recruitment and retention of high-quality students and faculty.

The acquisition of this instrument will stimulate inter-collegial communications at TU. We will continue to strengthen and reinforce our current collaboration between our TU forensic science faculty and the Maryland State Police department in providing research internships in forensic trace analysis for TU students, as well as to establish new collaborations with our neighboring institutions, namely Loyola University Maryland and Goucher College.

The research activities are based on interdisciplinary projects spanning the areas of condensed matter and materials physics, nanoscience, biology, geology, and analytical, inorganic, environmental, and forensic chemistry. The PI, Dr. Shields, is a biologist whose research focuses on insect ultrastructure, behavior, and physiology [1]. She has received past funding from NIH (AREA grant) to support her research and was previously the Director of the Office of Undergraduate Research at TU. Dr. Devadas, Co-PI, is a materials chemist with expertise in single particle optical spectroscopy and ultrafast laser spectroscopy. Dr. Smolyaninova, Co-PI, is a physicist studying metamaterials. Her expertise is in condensed matter, nanotechnology, and photonics. She is a past NSF Career grant awardee. Dr. Kolagani, Co-PI, is a condensed matter/materials physicist and studies perovskite metal oxide thin films. She serves as the program director for the Applied Physics Master's program and was a past recipient of the 2015 Wilson H. Elkins Professorship of the University System of Maryland. Dr. Williams, Co-PI, is a geobiologist investigating microbe-mineral biosignatures. She is currently involved in collaborations with the NASA Goddard Space Flight Center.

In this tutorial session, I will attempt to answer questions about submitting a Major Research Instrumentation (MRI) grant to the National Science Foundation (NSF). I will focus on what you should and should not do when submitting your grant application, who you should ask for letters of support, where you can obtain information about past submissions, forms, deadlines, etc., how to edit your submission if it is too long, and what you need from your institution for a successful application.

\section{References:}

[1] VDC Shields in "Microscopy: Science, Technology, Applications and Education”, eds. A. MéndezVilas and J.D. Diaz, (Formatex Research Center, Badojoz, Spain) vol. 1 (2010), p. 321-328.
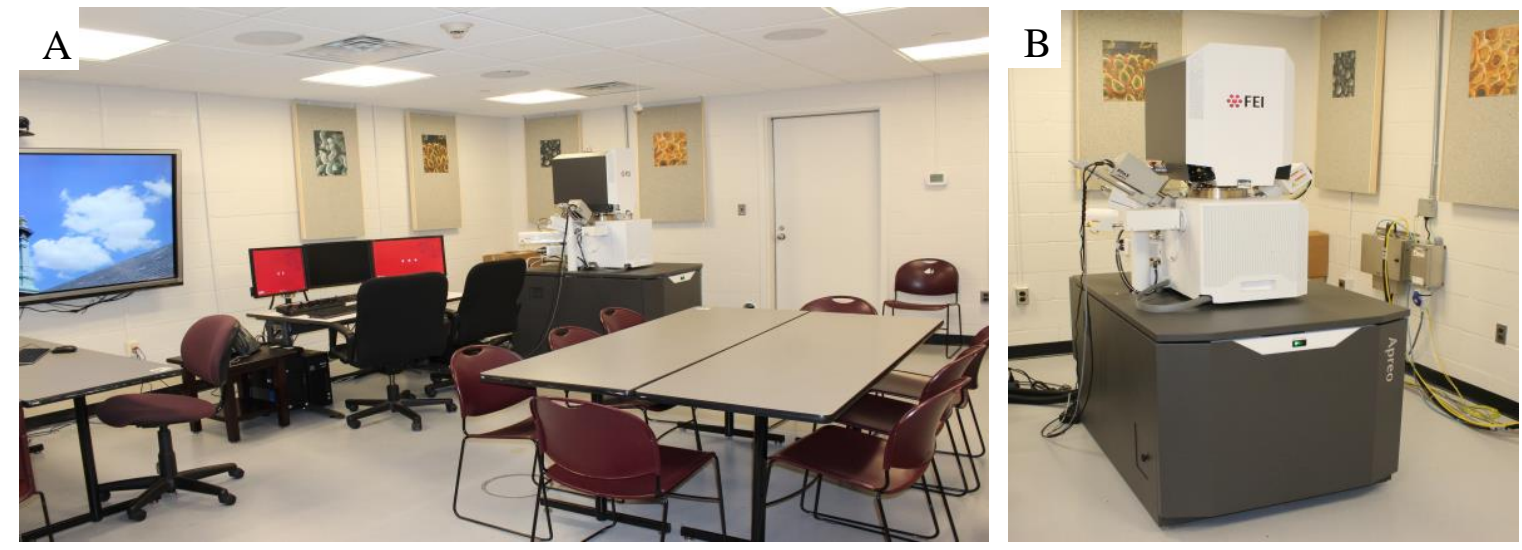

Figure 1 A. View of FESEM room at Towson University. B. Higher magnification view of FESEM. 